\title{
Energy efficiency evaluation method for submersible pushing- flow agitator for sewage treatment
}

\author{
Zhang Xiaoxin ${ }^{1}$, Huang Jin ${ }^{1},{ }^{*}$, Lin Ling ${ }^{1}$, Wang Yueping ${ }^{2}$, Zhang Xinheng ${ }^{2}$ \\ ${ }^{1}$ China National Institute of Standardization, 100191 Beijing, China \\ ${ }^{2}$ MCC HUATIAN Engineering \& Technology Corporation, 243011 Ma'anshan, China
}

\begin{abstract}
This paper introduced the development and research advance of the submersible pushing-flow agitator for sewage treatment at home and abroad. By analyzing four factors affecting the energy efficiency of the submersible pushing-flow agitator, the energy efficiency evaluation method and calculation formula of the submersible pushing-flow agitator for sewage treatment in China are analyzed and discussed in this paper.
\end{abstract}

\section{Introduction}

The submersible pushing-flow agitator for sewage treatment is the key equipment of mixing the sludge in sewage treatment process. By pushing-flow and mixing, the best flowing field and flow velocity of the sludge can be obtained without sedimentation and siltation. At the same time, the effect of aeration and mass transfer can be improved in the oxidation ditch. Since the submersible pushing-flow agitator continuously works in the sludge for years, its energy consumption is the third highest energy-consumption equipment in the sewage treatment plant besides aeration equipment and water pumps. In recent years, as Chinese enterprises began to absorb the foreign technology and invested numerous money to develop a series of submersible pushing-flow agitators, but each enterprise produced the agitators according to their own thinking, so the submersible push mixing market had no unified standard. In response to the "Energy Conservation Law of People's Republic of China", to enhance the energy utilization efficiency of the sewage treatment equipment, guide the progress of energy saving technology, optimize the product technical standards, standardize the sewage treatment equipment industry, and improve the international competitiveness of sewage treatment equipment in China, it is urgent to develop the scientific integrated energy efficiency evaluation method of sewage treatment submersible pushing-flow agitator.

\section{Submersible pushing-flow agitator for sewage treatment and its related standards}

According to the different speeds and main functions, the submersible pushing-flow agitators can be divided into three types, low-speed, medium-speed and highspeed submersible pushing-flow agitators. Generally, the installation position is about $4 \mathrm{~m}$ under the water. Its main functions include stirring, mixing and pushing flow that is stirring and mixing the sludge to form a certain flow and the flow field, without sedimentation and deposition. The submersible pushing-flow agitators for sewage treatment are widely used in the sewage treatment plants, and the energy efficiency will directly affect the cost of sewage treatment process.

The medium-speed and low-speed submersible pushing-flow agitator is composed of motor, deceleration mechanism, hydraulic component (paddle), static and static sealing components, lifting mechanism and electronic control system. The high-speed agitator has the same components accept retarder. The submersible pushing-flow agitator is a comprehensive, multidisciplinary equipment with wide technology coverage and high technology.

In the late 1970s, German ITT Company launched the world's first submersible pushing-flow agitator. After that, many foreign enterprises, such as Danish GRUNDFOS, Swiss Sulzer and the German ITT company, began to develop the submersible pushingflow agitator. At present, the widely used submersible pushing-flow agitator for sewage treatment in our country focuses on the so-called reliability and durability of the products, without considering the energy efficiency as the primary factor, and the type selection is not actively performed from the view of energy saving and consumption reduction. In fact, the energy efficiency and flow patterns of the imported products are not certified by any authoritative inspection institutions, the non-standard motors are widely applied, with high partsto-whole price ratio of the imported products, and no parts are available. At the very beginning, the submersible pushing-flow agitators in China were imported from foreign countries, until the middle of 1990 s, some enterprises began to develop submersible pushing-flow agitator. In the early stage of the

* Corresponding author: huangjin@cnis.gov.cn 
development of submersible pushing-flow agitator for domestic sewage treatment, the design of the submersible pushing-flow agitator for domestic sewage treatment is mostly based on the imitation. In recent years, with the development of the sewage treatment equipment industry in China, some enterprises and experts have started to research and manufacture the domestic sewage treatment submersible pushing-flow agitator. On the basis of introducing and absorbing the submersible pushing-flow agitator of Sweden Flygt company, Lin et al. discussed and studied the structure type selection, the main parameters determination, and the structural elements related to the hydraulic characteristics of the flow velocity and the main components (submersible motor, seal, protection device, etc.) of the submersible pushing-flow agitators[1]. Zhang et al. analyzed and compared the energy consumption and efficiency of the submersible pushing-flow agitators in some domestic and foreign sewage treatment equipment through the engineering application example, and proved the advantage of some domestic products compared with equivalents imported from foreign countries[2,3]. Zhao et al. discussed the optimization and improvement measures of the submersible pushing-flow agitators by optimizing the hydraulic performance of the impeller of the submersible agitator, improving the sealing and protection performance of the motor, and developing the best operation technique of the submersible agitator. After the test, the investment cost of the improved submersible agitator main equipment is reduced by over $30 \%$ compared with the equivalents in the international market, and the service life is increased by over $15 \%$ compared with the existing domestic equipment, and the operation cost is reduced by more than $5 \%$ in the meantime[4]. Tian et al. adopted 3D modeling software Pro/E to set up the entity model to study the influence of the submersible agitator distribution on the mixing effect in the sewage treatment tank, and provided reference for the practical engineering application of the submersible agitator [5]. Wang et al. explained the reference standards forthe model, installation and operation of the submersible agitator from the consumer's point of view in details, so that the consumers can follow this standard when selecting this equipment, to save the energy and reduce the consumption [6].

Before 2017, there was no national standard for submersible pushing-flow agitator in China whilst two industry standards were actually applied, which are the Specifications for environmental protection product Pusher submersible agitator (HJ/T279-2006) of the environmental protection industry and the Submersible agitator (CJ/T109-2007) of the urban and town construction industry. However, due to the limitations of the times of the development of industry standards, as well as the lack of research depth and awareness of the submersible pushing-flow agitator, design capacity and manufacturing process, the industry standards, in many aspects, were incomplete in the expression existed some shortcomings. No further progress have been made especially in terms of product safety, energy saving, consumption reduction, efficiency enhancement, and so on. Therefore, technical requirements and regulations can no longer satisfied the user's requirements of the technical performance of the product. With the increasingly stringent requirements of technological upgrading, energy saving and emission reduction, it is imperative to develop national standards to give true guidance to standardize the production and the manufacturing of the industry and the healthy competition and development of the industry.

The national standard Pusher Submersible agitator (GB/T33566-2017) which was officially released on May $12^{\text {th }}, 2017$, and formally implemented on December 1, 2017, marked the production, manufacturing, selection and the application of domestic-made pushing-flow agitator equipment would all have regulations to follow, and product quality traceability will be based on evidence. This national standard stipulates the classification and naming of submersible pushing-flow agitator, key components such as motors, electrical control equipment, deceleration mechanisms, impellers, dynamic and static sealing devices, housings, bearings, fasteners, lifting mechanisms, etc. Technical requirements, inspection methods and rules, labelling, packaging, transportation and storage are also involved. In particular, detailed and rigorous regulations have been made for the product safety, reliability, specific power performance and life of the submersible pushing-flow agitator. The standard have great maneuverability and foresightedness, which could give clear guidance and comprehensive analysis of the submersible pushing-flow agitator with high reliability, duration and energy efficiency, representing the vast majority of domestic submersible pushing-flow agitators. The design level and manufacturing strength of manufacturing companies also meet the needs of users as much as possible. Wang et al. analyzed and explained the main technical requirements and contents of the relevant national standards from the production and manufacturing of the submersible pushing-flow agitator. It helps the technicians engaged in the industry to understand and control the key points of the submersible pushing-flow agitator, and help the technicians who use the standard to design, process, manufacture, inspect and test to enhance the product quality control, providing basis for user design, selection, application and quality assurance [7].

\section{Factors affecting the energy efficiency of the submersible pushing-flow agitator}

For a long time, there is no energy efficiency standard neither special regulatory standard for the submersible pushing-flow agitator in China. So the production is made without supervision and regulatory basis. The manufacturers do not consider the difference of energy efficiency, whilst creating the vicious market competition with low price. So the products are significantly different in the shapes and structures, as well as the energy efficiency. 


\subsection{Influence of the hydraulic components (impeller) on energy efficiency}

The impeller is the key factor for the energy efficiency difference of the submersible pushing-flow agitator, even at the same speed or linear speed. If the impeller geometry, geometric accuracy, spiral angle, sweep angle, installation angle, symmetry balance and the force points are slightly different, it will cause direct and significant energy efficiency influence. The stirring, mixing and pushing flow effects are obvious, and there is no mixing blind area in the mixing pool. The sludge in the pool is not easy to precipitate with no sludge deposit. The impeller with small effective effect has poor working capacity. It can only stir, mix and push some water body in the pool and the sludge will precipitate and accumulate in the pool after several years.

\subsection{Influence of motor energy efficiency on product energy efficiency}

On one hand, the motor energy efficiency of different energy efficiency levels has various influence. For example, as for $5.5 \mathrm{~kW}$ 6-pole motor, if the energy efficiency is class 3 , the efficiency is $86 \%$ compared to $88 \%$ for class 2 energy efficiency, and $89.5 \%$ for class 1 . In addition, the motor efficiency with the same energy efficiency level but different poles varies greatly. For example, as for $5.5 \mathrm{~kW}$ motor with class 2 energy efficiency, the efficiency of 4-pole motor is $89.6 \%$, while 6-pole motor is $88 \%$ and 12 -pole motor is $70 \%$.

\subsection{Influence of transmission efficiency of deceleration mechanism on product energy efficiency}

The transmission efficiency of the deceleration mechanism is different when adopting different types of deceleration transmission. For example, the cycloid needle wheel deceleration mechanism is not applicable for $24 \mathrm{~h}$ continuous operation, the long-term wearing will easily cause skidding, the transmission efficiency is significantly reduced, and the retarder life time will not exceed 2 years when it operates continuously.

\subsection{Influence of structure design on product energy efficiency}

Minor differences in the product structure design will lead to significant difference in the product performance, such as the influence of mechanical seal and bearing matching size. If the interference amount of the mechanical seal is increased, the power consumption will be greatly increased.

In the factors above, the impeller of hydraulic parts will provide the greatest influence, but other factors cannot be ignored, because the difference in efficiency of each factor accumulates continuously and amplifies continuously, resulting in obvious difference in the energy efficiency.

\section{Energy efficiency evaluation methods for submersible pushing-flow agitator}

The concept of "thrust----power ratio" is proposed in BS ISO 21630 " Pumps - Testing — Submersible mixers for wastewater and similar applications" [8]. The submersible pushing-flow agitator is applied to the sewage treatment process to prevent the sludge from deposition and silting. In order to achieve this goal, the mixture of sludge and water must be fully stirred and mixed, and then pushed forward, the sludge cannot stagnate in the effective flow field only in this way. The traditional concept of "thrust---power ratio" considers that "whichever agitator is used, the final output is thrust", but the thrust must be achieved by the impeller rotation, and the force is a vector with directionality. The force of the submersible pushing-flow agitator impeller is not only the axial force, but also the circumference force (or called as the cut force), in which the axial thrust is to make the sludge mixture flowing forward with a special flow state at a certain flow velocity, and the circumferential force is to fully stir and mix the water at three levels and form flow patterns of upper and lower circulation in the flow field. The two flow states form 3D complex flow pattern. If considering only the thrust according to ISO21630 standard while neglecting the circumferential force, the thrust power value is smaller, and it can't reflect the energy efficiency of the equipment accurately. The standard requirement of the flow velocity $0.3 \mathrm{~m} / \mathrm{s}$ can be easily obtained since higher thrust is required, thus the compound function and effect of stirring and mixing is neglected, forming undesirable flow state and field. So it is impossible for the sludge at the bottom of the tank to mix while flowing.

On the other hand, the "thrust---power ratio" is suitable for the performance requirements of the ship spiral propeller since the main role of the propeller is to generate the axial thrust to make the heavy-load ship driving, the ship spiral propeller wing is design with concept of " maximum driving force with the minimum energy consumption", and it is normal if the wave is promoted during the process. However, different from this, if the submersible pushing-flow agitator is used in the anoxic pool, the sewage shall not roll; otherwise, it will cause the aeration in the anoxic pool due to the fluctuation of water, losing the function and effect of anoxic acidification.

In conclusion, the "thrust--power ratio" proposed in BS ISO 21630 is referable for evaluating the performance of submersible pushing-flow agitator, but it is not recommended to use the parameter as the energy efficiency parameter of the submersible pushing-flow agitator. Referring to the same product standard for the fluid machinery category, with similar working form and condition to this submersible pushing-flow agitator, the standard of "Minimum allowable values of energy efficiency and energy efficiency grades of AC electric fans"(GB12021.9-2008) stipulates that the minimum allowable energy efficiency unit of AC fan is $\mathrm{m}^{3} / \mathrm{min} \cdot \mathrm{w}$ in the standard test conditions. "The air volume produced by unit energy consumption" is taken as the index of 
energy efficiency evaluation. Therefore, referring to GB12021.9-2008, and keeping the principle consistent with GB50014 "Code for design of outdoor wastewater engineering", the "specific power" defined in GB/T33566 "Pusher submersible agitator" (in the specified test conditions, the total power consumed at the flow rate at least $0.3 \mathrm{~m} / \mathrm{s}$ of water body in a cubic meter) is used as the energy efficiency evaluation index of the submersible pushing-flow agitator. Calculation formula is shown in equation (1):

$$
\mathrm{E}=\frac{\mathrm{P}}{\mathrm{V}}
$$

Where: E -Specific power, $\left(\mathrm{W} / \mathrm{m}^{3}\right)$;

$\mathrm{P}$ - Motor input power, (W);

$\mathrm{V}$ - Equivalent effective volume, $\left(\mathrm{m}^{3}\right)$.

\section{Acknowledgment}

This work was supported by the Foundation of President of China National Institute of Standardization (542018Y5926) and National Key R\&D Program of China (2018YFF0213201).

\section{References}

1. Lin Zheng. MechanicalDdesign and Research, (4): 17-20 (1994).(in chinese)

2. Zhang Xinheng, QianWeixia. Industrial Water Treatment, 27 (1): 87-89 (2007). (in chinese)

3. Zhang Xinheng, QianWeixia. China Environmental Protection Industry, (12): 28-30 (2006). (in chinese)

4. Zhao Xuelian, Tang Di, Cao Dajun, et al. Newborn Baby ,21 (2014). (in chinese)

5. TianFei, Shi Weidong, Lu Xining, et al. Journal of Drainage and Mechanical Engineering, 31 (2): 146150 (2013). (in chinese)

6. Wang Chengduan, Liao Yi. Advances in Environmental Protection, 04 (2): 17-21(2014). (in chinese)

7. Wang Yueping, Wang Qiujing, Wang Xun. China Environmental Protection Industry, (11): 46-48 (2017). (in chinese)

8. BS ISO 21630: Pumps - Testing - Submersible mixers for wastewater and similar applications. 2007. 